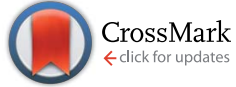

Cite this: RSC Adv., 2015, 5, 54985

Received 5th May 2015

Accepted 15th June 2015

DOI: $10.1039 / c 5 r a 08317 f$

www.rsc.org/advances

\section{Thin film composite polyamide membranes: parametric study on the influence of synthesis conditions}

\begin{abstract}
B. Khorshidi, ${ }^{a}$ T. Thundat, ${ }^{\text {b }}$ B. A. Fleck ${ }^{a}$ and M. Sadrzadeh ${ }^{\star a}$
Preparation of thin film composite (TFC) polyamide (PA) membranes by interfacial polymerization (IP) reaction is remarkably sensitive to the interactions between synthesis parameters. Here we report the effect of the simultaneous change in four synthesis parameters, namely monomers concentrations ( $m$-phenylenediamine, MPD, and trimesoyl chloride, TMC), reaction time and curing temperature, on the surface morphology and on the permeation properties of TFC membranes. By varying several synthesis parameters at the same time using a Taguchi robust design (L9 orthogonal arrays), it was found that monomers concentration and curing temperature significantly affected water permeation by creating a substantial change in morphology of the PA films. More importantly, a strong interaction between monomers concentration was observed, which demonstrates the importance of smart adjustment of these parameters in the preparation process. Permeation properties were justified by thickness and by the cross-link density of the synthesized films; the latter was found to be more influential. Based on analysis of variance (ANOVA), the contribution of the synthesis parameters towards change in water permeation was determined as: curing temperature $(40.7 \%)>$ MPD concentration $(28 \%) \sim \mathrm{TMC}$ concentration $(27.8 \%)>$ reaction time $(1.9 \%)$. The findings will provide valuable guidelines to develop practical low cost, robust and high performance membranes by changing the curing temperature and the monomer concentrations as critical parameters.
\end{abstract}

\section{Introduction}

Over the past decades, membrane filtration processes have attracted great attention in desalination and water purification applications. ${ }^{1,2}$ Currently, most commercial reverse osmosis (RO) and nanofiltration (NF) membranes are made in the form of thin film composites (TFC)s which have an ultrathin polyamide (PA) barrier film $(\sim 200 \mathrm{~nm})$ over a polysulfone (PSf) or polyethersulfone (PES) intermediate layer $(\sim 50 \mu \mathrm{m})$ and a polyester fabric bottom layer $(120 \mu \mathrm{m})$. The PA film is often synthesized by an interfacial polymerization (IP) reaction between polyfunctional amine and acyl chloride monomers at the surface of a porous sublayer. ${ }^{3,4}$ Demands to improve the productivity of the composite membranes have triggered much research to understand and optimize the properties of the top layer which is responsible for the final productivity and selectivity of these types of membranes. A brief overview of earlier studies on the influence of synthesis conditions on the structure and transport properties of TFC

${ }^{a}$ Department of Mechanical Engineering, University of Alberta, 6-074 NINT Building, Edmonton, AB T6G 2G8, Canada. E-mail: sadrzade@ualberta.ca; Fax: +1 (780) 492 2200; Tel: +1 (780) 4929099

${ }^{b}$ Department of Chemical and Material Engineering, University of Alberta, 7-026 ECERF, Edmonton, AB T6G 2V4, Canada membranes is presented in Table $1 .^{5-14}$ According to this table, the literature experimental results do not form a clear consensus about the trend of influence of the synthesis parameters on the final properties of the TFC PA membranes. Therefore, further research is needed in order to produce definitive practical conclusions leading to the robust and high performance membrane designs. Particular research challenges in this field are (i) to fundamentally and systematically understand the effects of reaction conditions on the characteristics of fabricated thin films, and (ii) to identify the most influential parameters affecting transport properties of the membranes.

The studies reviewed in Table 1, suffer from the limitation that the synergistic effects of synthesis parameters were not investigated as most experiments were conducted using the one-factor-at-a-time (OFAT) method where only one factor (variable) was changed at each experimental trial. Although OFAT leads to clearly expressed conclusions, the chance of missing optimum conditions and not observing the covariance between parameters is high in this approach. ${ }^{15,16}$ Thus, existing results should be interpreted with caution, and the widely accepted hypotheses for the effect of synthesis conditions require further revaluation to be extrapolated to novel practices. 
Table 1 Overview of experimental studies regarding the effects of synthesis conditions on performance of TFC membranes

\begin{tabular}{llll}
\hline Reference & Studied parameters & Experimental strategy & $\begin{array}{l}\text { Range of performance } \\
\text { Flux }\left(\mathrm{L} \mathrm{m}^{-2} \mathrm{~h}^{-1} \mathrm{bar}^{-1}\right)\end{array}$ \\
\hline $\begin{array}{l}\text { Chai and Krantz, } \\
1994 \text { (ref. 5) }\end{array}$ & $\begin{array}{l}\text { Monomertion(\%) concentration, } \\
\text { reaction time }\end{array}$ & OFAT & $\underline{0.39-0.74}$ \\
\hline
\end{tabular}

Rao et al., 1997 (ref. 6)

Rao et al., 2003 (ref. 7)

Song et al., 2005 (ref. 8)

Roh et al., 2006 (ref. 9)

Ghosh et al., 2008 (ref. 10)

Liu et al., 2008 (ref. 11)
Reaction time, curing time and temperature

Reaction and curing time and temperature

Monomers concentration, reaction time

Monomers concentration

Organic solvent properties, curing time and temperature

Reaction time, $\mathrm{pH}$ of water solution, curing time and temperature
OFAT

$\frac{0.78-1.17}{75-97}$

OFAT

$\frac{0.68-1.33}{74-97.5}$

Not specified

OFAT

$\frac{0.3-2}{50-98}$

Not specified

$\frac{0.76-1.8}{93-98}$

OFAT

$\frac{0.38-0.7}{64.6-99.4}$
Major results

- PA thickness increased with increase in TMC

concentration and reaction time, but did not change when MPD concentration increased

- Water flux dramatically decreased with increase in reaction time

- Flux and rejection increased with increase in reaction time up to $60 \mathrm{~s}$, but rejection decreased dramatically for higher reaction time

- Increasing curing temp. up to $75{ }^{\circ} \mathrm{C}$ improved both flux and rejection, higher curing temperature lowered the flux substantially

- Decreasing the reaction time and curing temperature resulted in thinner membranes with higher water flux

- Salt rejection was reported to be insensitive to modified conditions

- Permeation flux increased with increase in reaction time at low TMC

concentration; the opposite trend was reported at high

TMC concentration

- Increasing MPD

concentration improved salt rejection but lowered the water flux

- PA film thickness increased with increase in both MPD and TMC concentrations - Surface hydrophilicity increased by increasing TMC concentration, but decreased as MPD concentration increased - High surface tension, low viscosity solvents led to higher permeation performance for membranes - Flux and rejection increased with increase in curing temperature

- Increasing reaction time reduced permeation flux and improved salt rejection - Water permeation increased with increase in TMC concentration 


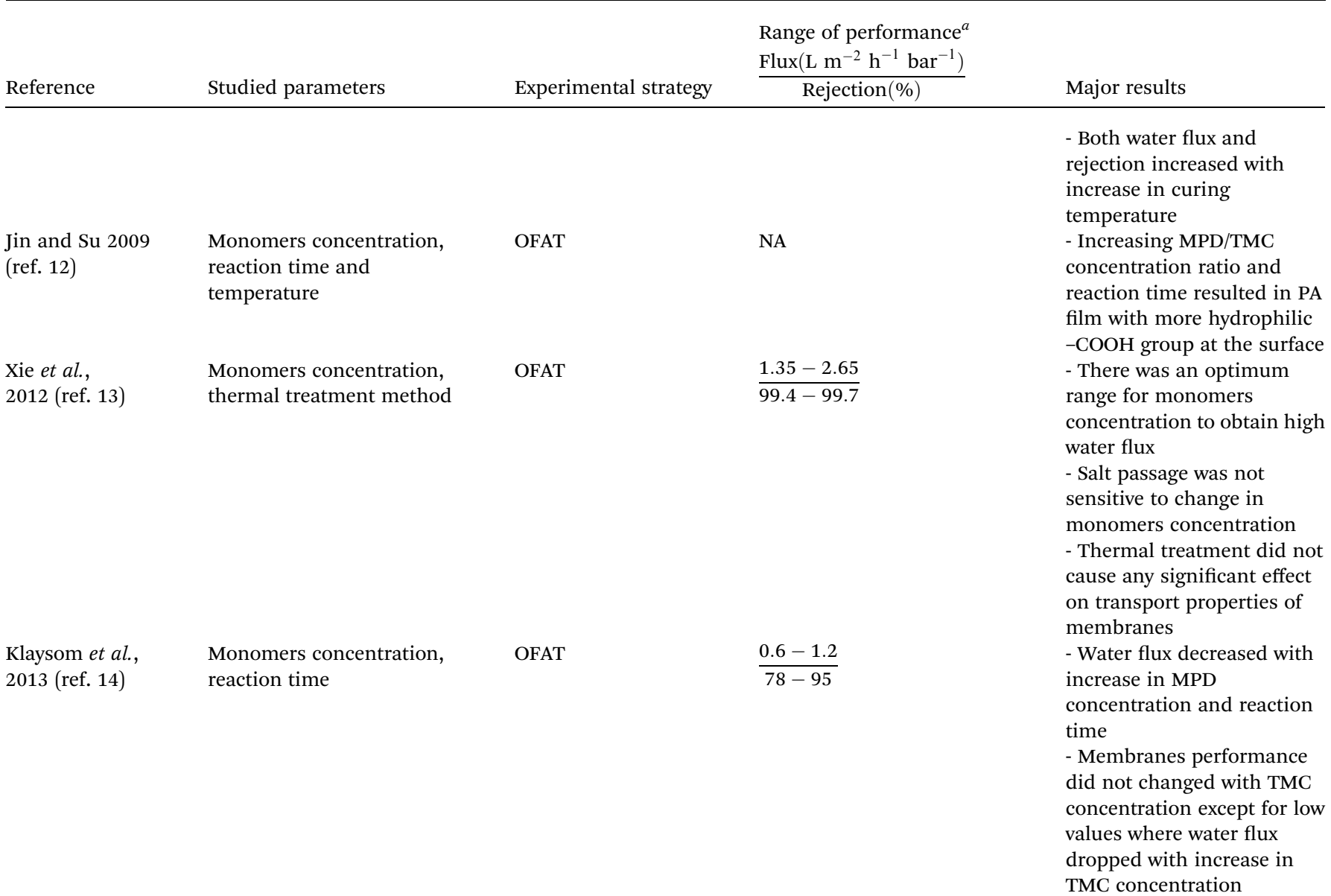

${ }^{a}$ All reports used RO test cell to study membranes performance except ref. 12 which only used Fourier transform infrared (FTIR) spectroscopy for membrane characterization. ${ }^{b}$ Reacting monomers are $m$-phenylenediamine (MPD) and trimesoyl chloride (TMC) for all studies except ref. 11 which used 5-chloroformyloxyisophthaloyl chloride (CFIC) in organic solution.

In the current work, we present a systematic study of the effect of simultaneous changes of synthesis conditions on surface morphology, and permeation properties of TFC membranes. Four important synthesis parameters, namely monomer concentration, reaction time and heat curing temperature were considered as influential factors. The experimental trials were arranged using a robust design of experiment (DOE) method known as Taguchi to minimize the number of experiments while quantifying the significance of each parameter and obtaining valuable information about the interrelationship between the parameters. The results obtained by the Taguchi method were verified by synthesizing additional confirmation membranes and linking the change in permeation properties to the alteration of membrane morphology. A detailed interaction analysis of monomers concentrations was conducted to find ranges of concentrations for which synthesized membranes did not follow the predicted behaviour. The interaction analysis provided valuable guidelines for fabricating a range of salt rejecting membranes from loose NF to tight RO.

\section{Materials and methods}

\section{Chemicals and reagents}

Microporous polyethersulfone (PES) $(0.2 \mu \mathrm{m})$ was provided by Sterlitech Co. (WA, USA) and was used as the support material in the composite membranes. m-Phenylenediamine (MPD, $\geq 99 \%$ ), trimesoyl chloride (TMC, 98\%) and camphorsulfonic acid (CSA) were obtained from Sigma-Aldrich. Cyclohexane $(\geq 99 \%$ ), triethylamine (TEA) and sodium dodecyl sulphate (SDS) were purchased from Fisher Scientific. All materials were used as they were received from suppliers.

\section{Synthesis of thin film composite (TFC) membranes}

The thin film composite membranes were prepared by IP reaction between MPD in water and TMC in cyclohexane at the surface of the PES substrate. The PES support was first immersed in MPD solution with 2 wt $\%$ CSA, 1 wt $\%$ TEA and $0.2 \mathrm{wt} \%$ SDS for 10 minutes. TEA was used as an acylation catalyst to promote the IP reaction by removing $\mathrm{HCl}$ which is formed as a by-product of the IP reaction. SDS and CSA are used 
Table 2 Control factors and their corresponding levels

\begin{tabular}{lllll}
\hline \multicolumn{5}{c}{ Controllable factors } \\
\cline { 2 - 5 } Run & MPD concentration (wt\%) & TMC concentration (wt \%) & Reaction time (s) & Curing temperature $\left({ }^{\circ} \mathrm{C}\right)$ \\
\hline M1 & 1 & 0.15 & 15 & 25 \\
M2 & 1 & 0.25 & 30 & 55 \\
M3 & 1 & 0.35 & 60 & 85 \\
M4 & 1.5 & 0.15 & 30 & 85 \\
M5 & 1.5 & 0.25 & 60 & 25 \\
M6 & 1.5 & 0.35 & 15 & 55 \\
M7 & 2 & 0.15 & 60 & 55 \\
M8 & 2 & 0.25 & 15 & 85 \\
M9 & 2 & 0.35 & 30 & 25 \\
\end{tabular}

to improve the absorption of MPD solution into the PES support. $^{10}$ The concentration of these additives was kept constant in all solutions. The substrate was then removed and the excess amine solution was squeezed off the surface using a rubber roller. Next, the impregnated PES sheet was brought into contact with TMC solution to allow a polymerization reaction at the surface at different reaction times. The resulting composite membranes were then cured in digital oven for 5 minutes at various curing temperatures. Finally, to remove any residual solution from the surface, the membranes were washed 10 times with $250 \mathrm{~mL}$ deionized (DI) water and then kept in the DI water bath until characterization tests were performed.

\section{Experimental methodology}

The experimental matrix was designed using the Taguchi method which invokes orthogonal arrays (OA) of factors to study the simultaneous influence of several variables on a target response by conducting a minimum number of experiments. ${ }^{17}$ The Taguchi method employs plots of "marginal means" which give useful information about the trend of factor influence on the response as well as the response sensitivity to a change in a factor. ${ }^{18}$ Moreover, the response variability due to signal (controllable) and noise (uncontrollable) factors can be measured by calculating the signal to noise (SN) ratio. This parameter is used to optimize the factors combination and to consequently minimize the response sensitivity to noise factors. The method of calculation of the SN ratio depends on the goal of experiment. There are three standard SN ratios, namely larger-the-better, smaller-the-better and nominal-the-best. ${ }^{\mathbf{1 7}, 18}$ For all these cases, a higher value of SN ratio suggests better quality of the product and lower variation in the response due to random noise factors. The following equation was used to calculate the larger-the-better SN ratio in the present work:

$$
\mathrm{SN}=-10 \log \left(\frac{1}{n} \sum_{i=1}^{n} \frac{1}{y_{i}^{2}}\right)
$$

where $n$ is the number of experiments and $y_{i}$ is the experimental result (response) at each trial.

The general steps of applying the Taguchi method are (i) determining the quality characteristic of interest and designing the OAs matrix of experiments by identifying the controllable factors and their corresponding levels, (ii) running the designed experiments and analysing data by determining the average factor effects, and (iii) conducting confirmation experiments to verify the extracted trends and predicted results. The control variables investigated in the present study and their corresponding levels and arrangement in the L9 (4 three-level factors) array are presented in Table 2. Each run was replicated twice (18 membranes were synthesized in total) to evaluate the repeatability of membrane formation.

\section{Characterization of PA membranes}

The surface morphology of TFC membranes was studied using field emission scanning electron microscopy (FESEM, JEOL $6301 \mathrm{~F}$ ). All membranes were sputter coated with a thin film of chromium and imaged at a magnification of $30 \mathrm{k} \times$. The ultrathin cross-sections were stained in uranyl acetate and lead citrate, embedded in spurrs resin, sectioned using Ultramicrotome (Reichert-Jung Ultracut E) and examined by Philips/FEI (Morgagni 268) transmission electron microscope (TEM) at an acceleration voltage of $80 \mathrm{kV}$. The functional groups present at the surface of the PA membranes were investigated using attenuated total reflectance-Fourier transform infrared (ATR-FTIR) spectroscopy. The ATR-FTIR microscope (Thermo Nicolet Nexus 670, USA) was equipped with a mercurycadmium-tellurium (MCT) detector. The spectrum of each membrane was averaged from 512 scans and collected over the range of $600-4000 \mathrm{~cm}^{-1}$ at $4 \mathrm{~cm}^{-1}$ resolution.

$\mathrm{X}$-ray photoelectron spectroscopy (XPS) is used for analyzing elemental composition $(\mathrm{C}, \mathrm{O}, \mathrm{N})$ for the top 1-10 $\mathrm{nm}$ of the membranes surface. The TFC membranes examined using a Kratos AXIS ULTRA spectrometer equipped with a monochromatic $\mathrm{Al} \mathrm{K} \alpha \mathrm{X}$-ray source. The source was run at a power of $210 \mathrm{~W}(14 \mathrm{~mA}, 15 \mathrm{kV})$ using a hybrid lens with a spot size of $700 \mu \mathrm{m} \times 400 \mu \mathrm{m}$. Survey spectra were collected with a pass energy of $160 \mathrm{eV}$, step size of $0.4 \mathrm{eV}$, and sweep time of $100 \mathrm{~s}$ in the range of $0-1100 \mathrm{eV}$. High resolution spectra for $\mathrm{C}, \mathrm{O}$ and $\mathrm{N}$ elements were collected with pass energy of $20 \mathrm{eV}$, step size of $0.1 \mathrm{eV}$, and sweep time of $200 \mathrm{~s}$.

The permeation properties of the TFC membranes were evaluated using a crossflow membrane filtration system used previously. ${ }^{19}$ The performance measurements were carried out 
at a trans-membrane pressure of $1.52 \mathrm{MPa}$ and at a feed flow

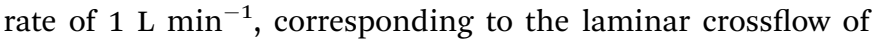
Reynolds number $\mathrm{Re}=730$.

Water flux $\left(J_{\mathrm{w}}\right)$ at steady state was obtained by measuring the volume of water $(\Delta V)$ passed through the filtration surface $(A)$ during the specific time period of sample collection $(\Delta t)$ :

$$
J_{\mathrm{W}}=\frac{\Delta V}{A \Delta t}
$$

The apparent salt rejection was calculated by measuring the concentration of salt in the permeate after $3 \mathrm{~h}$ filtration of 2000 ppm $\mathrm{NaCl}$ solution at $25{ }^{\circ} \mathrm{C}$ as follows:

$$
R=\left(1-\frac{C_{\mathrm{p}}}{C_{\mathrm{f}}}\right) \times 100
$$

where $C_{\mathrm{p}}$ and $C_{\mathrm{f}}$ are the $\mathrm{NaCl}$ concentration in the permeate and feed solutions, respectively. ${ }^{20}$ The salt concentration in each solution was obtained based on a calibration curve of solution conductivity.

\section{Results and discussion}

\section{Surface characterization results}

The chemical composition of prepared membranes is analysed by ATR-FTIR and the spectra are presented in Fig. 1 over the wave numbers of $1350-1750 \mathrm{~cm}^{-1}$. The spectra of bare PES support showed three peaks at 1410, 1485 and $1580 \mathrm{~cm}^{-1}$ due to presence of aromatic ring (benzene) skeletal vibration. ${ }^{21,22}$ These peaks were also identified in all synthesized membranes due to high penetration depth of IR beam $(>300 \mathrm{~nm})$ at this region (1350-1750 $\left.\mathrm{cm}^{-1}\right)$. However, the three new peaks at 1541 , $1611,1667 \mathrm{~cm}^{-1}$ in the spectra of the TFC membranes revealed the presence of interfacially polymerized PA film at the surface of the PES support. These peaks were associated with $\mathrm{C}=\mathrm{O}$ stretching of amide I bond, aromatic amide ring breathing and $\mathrm{N}-\mathrm{H}$ bending of amide II in -CO-NH- group, respectively. ${ }^{22}$ According to Fig. 1, the similar spectra characteristics of the

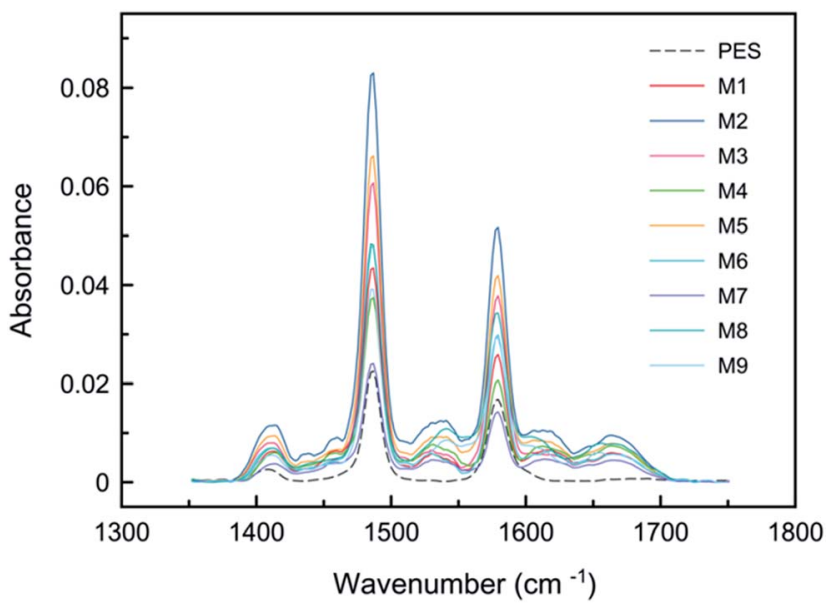

Fig. 1 ATR-FTIR spectra of TFC membranes.
TFC membranes suggest the presence of PA layer with similar basic structure in spite of different synthesis conditions.

\section{Permeation performance}

Fig. 2 presents the average water flux and salt rejection of the synthesized TFC membranes (M1-9). As it is shown in this figure, the water flux and salt rejection of all membranes were in a mutually exclusive relationship, so that when the water flux increased, the salt rejection decreased and vice versa. While the rejection percentage of the membranes varied in the range of 94-98\% the water flux changed remarkably in the range of 7-68 $\mathrm{L} \mathrm{m}^{-2} \mathrm{~h}^{-1}$ (LMH) which indicates a greater influence of synthesis parameters on water permeability leaving the rejection rate at consistently high and desirable value.

The amine/acyl chloride $\left(-\mathrm{NH}_{2} /-\mathrm{COCl}\right)$ molar ratio in Fig. 2 is a criterion showing the amount of reactive functional groups present in polymerization reaction. This ratio is calculated by converting the initial mass concentration of MPD and TMC in their corresponding solvents to molar concentration considering the numbers of $-\mathrm{NH}_{2}$ and $-\mathrm{COCl}$ functional group in each monomer (two $-\mathrm{NH}_{2}$ and three $-\mathrm{COCl}$ groups). According to Fig. 2, the membranes with low molar ratio $\left(-\mathrm{NH}_{2} /-\mathrm{COCl}<7\right)$ showed significantly different flux/rejection behaviour compared to other membranes. As will be shown later, very high molar ratio $\left(-\mathrm{NH}_{2} /-\mathrm{COCl}>30\right)$ also changes the separation performance of composite membranes considerably. The $-\mathrm{NH}_{2} /-\mathrm{COCl}$ molar ratio directly affects the rate of monomers diffusion and polymerization reaction at the surface, which subsequently changes the resulting morphology and water permeation of membranes. The detailed explanation of the effect of monomer concentration on the properties of synthesized PA films is discussed in the following section.

\section{Effect of monomer concentration}

The plot of "marginal means" for water flux, salt rejection and their corresponding SN ratios at different levels of each control factor are shown in Fig. 3 and 4. The marginal graphs are

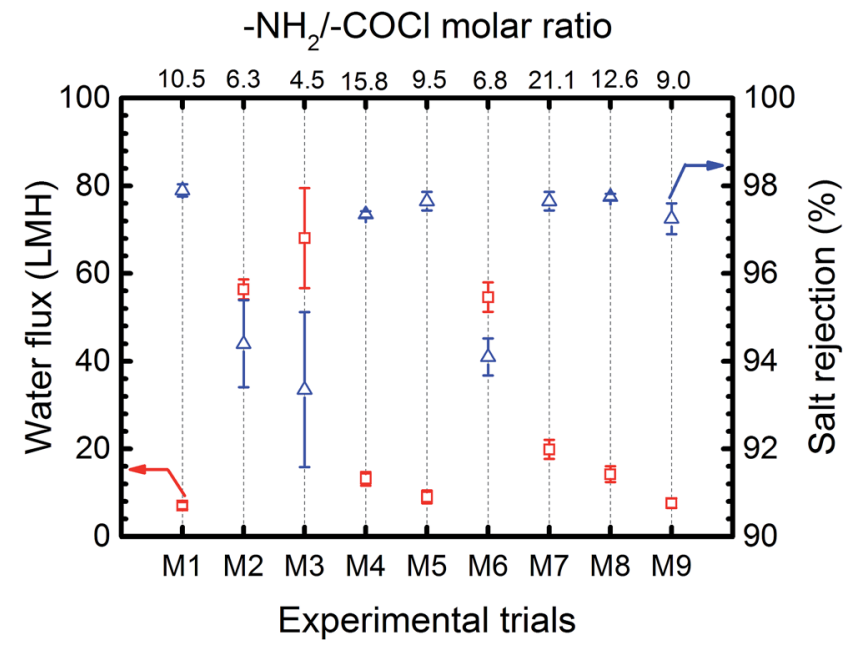

Fig. 2 Water flux and salt rejection of all synthesized membranes. 
plotted by averaging the results of experimental trials at a specified value of a parameter. For example, the mean water flux for the membranes made with $1 \mathrm{wt} \%$ MPD concentration is the average of the results of M1 to M3 where MPD concentration is 1 $w t \%$. Although more than one factor is varying at each run of Table 2, the arrays orthogonality in this table allows an independent estimation of the influence of each factor on the response. Fig. 3a and 4a show that the average influence of increasing MPD concentration in the aqueous solution was the formation of TFC membranes with higher salt rejection but lower water flux. Exactly the opposite trend was observed by increasing the TMC concentration as indicated in Fig. $3 \mathrm{~b}$ and 4 b. SN ratios in these figures account for response variability, so that the higher the SN ratio the lower the variability of the response, thus the better the quality of the product. According to Fig. 3 and 4, the mean responses and SN ratio followed the same trend, implying that the optimum condition would also provide the least variability in response and maximum robustness. Furthermore, the relatively high $\mathrm{SN}$ ratios in these graphs show that the levels of control factors were adjusted properly so that the effects of the uncontrollable or unknown noise factors on the response were minimized.

The extracted trends using Taguchi marginal graphs can be verified by comparing the performances of membranes synthesized by only changing the levels of one specific factor when all other factors maintained constant. Since no two runs in the L9 matrix are comparable, 11 extra confirmation membranes were prepared at combinations, which were not considered in Table 2. The synthesis conditions and permeation performance of these confirmation membranes are presented in Table 3. Taguchi predicted flux and rejection in this table are calculated by Taguchi transfer function (a linear model) that will be explained in ANOVA section. By comparing the permeation results of $\mathrm{C} 1$ vs. $\mathrm{C} 2$ and $\mathrm{C} 3$ vs. $\mathrm{C} 4$, it is confirmed that membranes prepared at higher MPD concentration showed lower water flux and higher salt rejection.

In order to explain the observed trends in Fig. 3 and 4, the mechanism of PA formation via interfacial polymerization should be understood. The IP reaction initiates at the surface when the MPD/water impregnated PES support is brought into contact with TMC/organic solvent solution. Since water and organic solvent are immiscible, the polymerization reaction occurs at the water/organic interface. ${ }^{23-25}$ In most IP reactions, the MPD concentration in water is much larger than the TMC concentration in the organic solvent, due to negligible solubility, vigorous reaction of the acyl chloride monomer with water and good solubility of amine in organic solvent. ${ }^{5,10}$ Hence, the IP reaction is believed to be mainly controlled by diffusion of MPD molecules to the reaction zone and thus the polymer film grows from the water/organic interface toward the bulk of the organic solution. ${ }^{26,27}$ However, the growth of the PA film does not follow a linear increase over the reaction time. Immediately after the contact of the two monomers solution the water/organic interface provides an empty platform for fast reaction of MPD and TMC molecules to form an ultrathin incipient (core) PA film at the PES surface. After that, polymerization shifts to a slow growth stage where the MPD diffusion and thus reaction rate slows down significantly due to
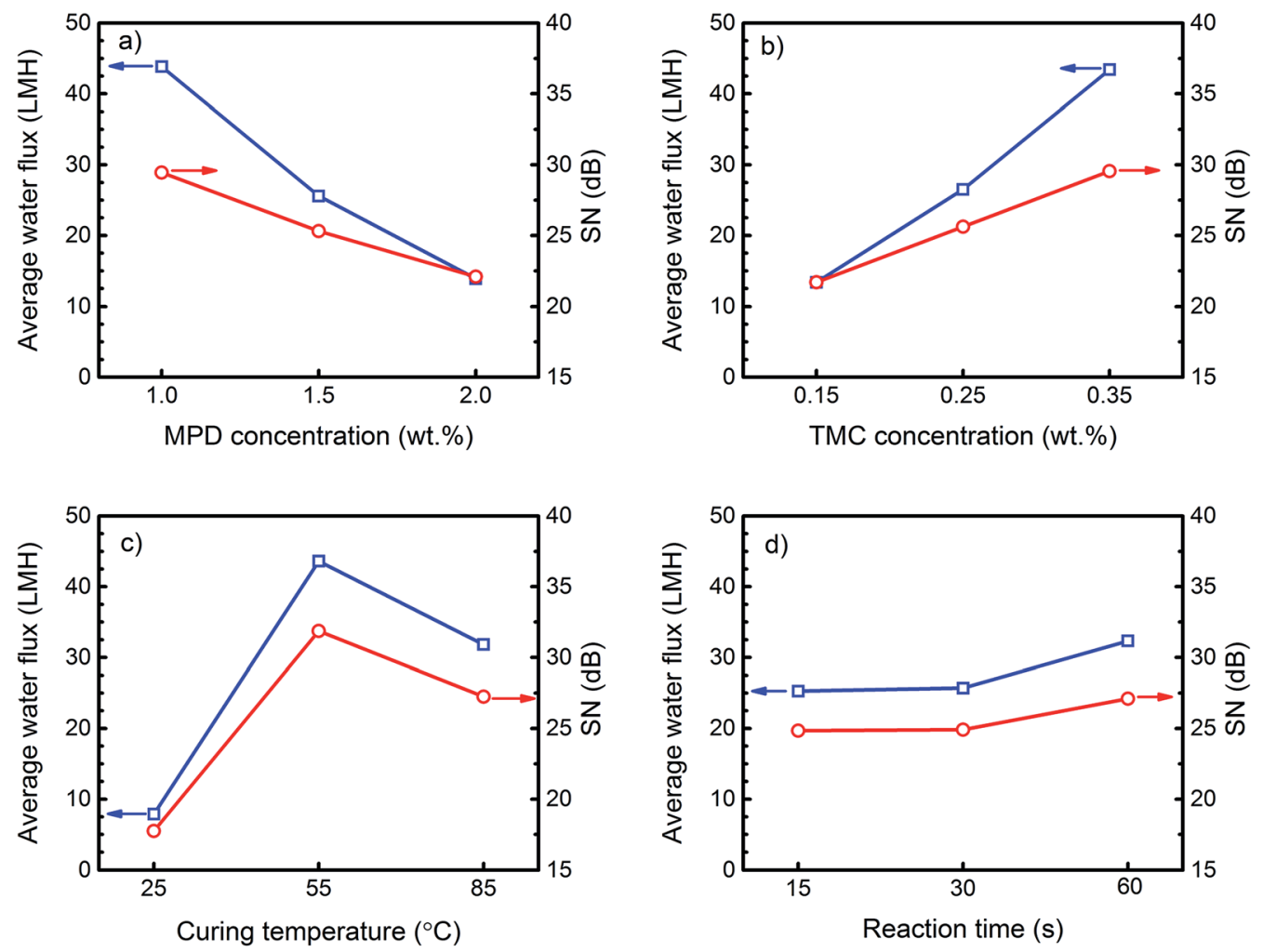

Fig. 3 Average water flux and SN ratio for each level of each control factor. 

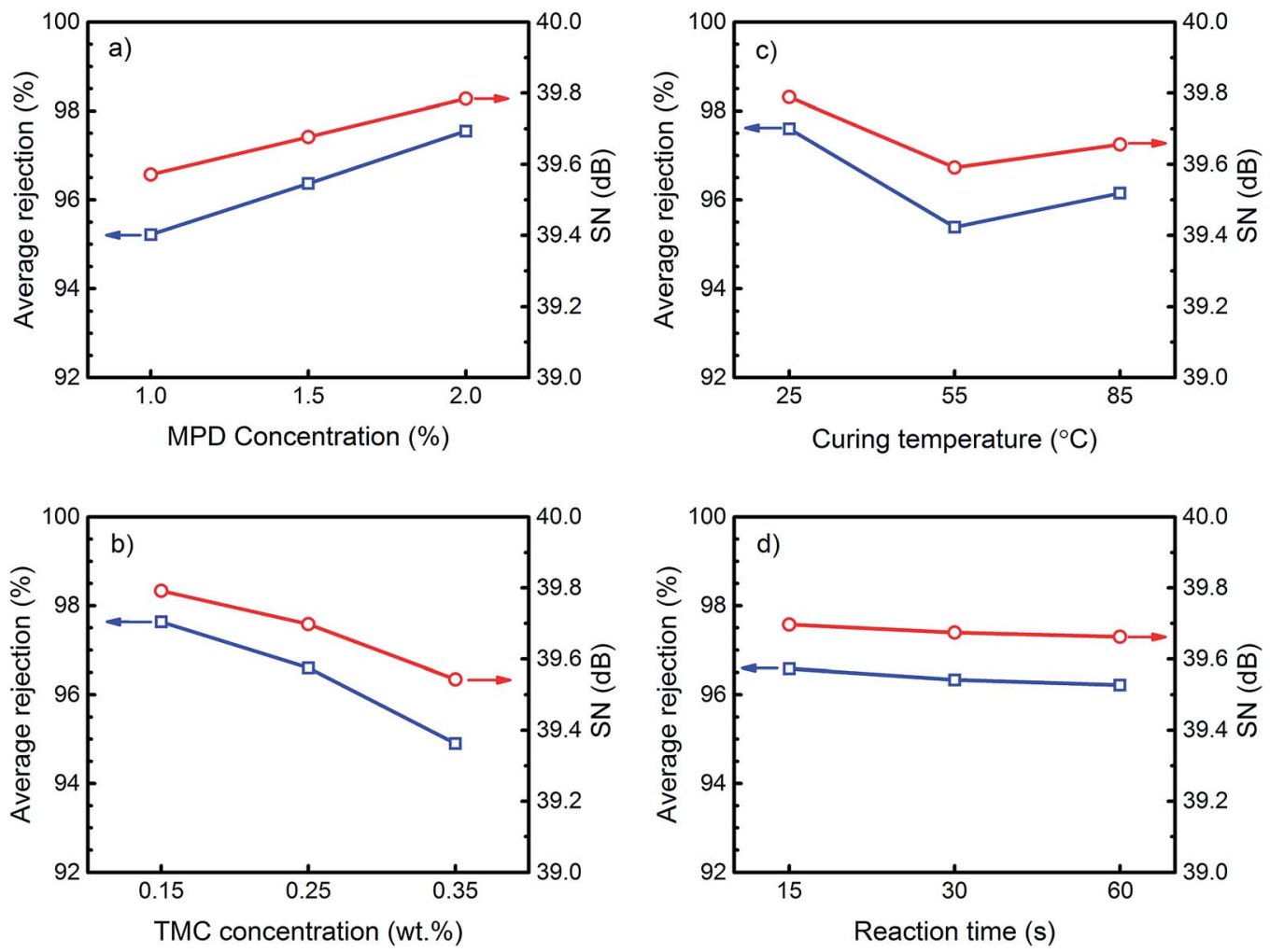

Fig. 4 Average salt rejection and SN ratio for each level of each control factor.

presence of the incipient (core) layer. ${ }^{28}$ The result of polymerization at this stage is the formation of a second layer with the so-called ridge-and-valley morphology which covers the core layer. ${ }^{10,13,27}$ The separation performance of the final PA layer depends not only on the overall film thickness (the thicker the film is, the lower the water permeates), but also on the crosslink density of the internal structure. ${ }^{13}$ It is worth mentioning that cross-link density of polymer corresponds directly with its crystallinity. The crystallinity is primarily determined by the chain flexibility and the volume fraction of polymer. The transport mechanism through a dense membrane like the most of polyamide TFC RO membranes is by the molecular diffusion of permeants through the free volume elements between the polymer chains which are varying due to thermal motion of the chains. ${ }^{29}$ Hence, the crystallinity can significantly affect the water permeability by varying the space between the polymer chains (the higher the crystallinity the lower the permeation flux). ${ }^{30}$ The polyamide active layer in TFC membranes is a semicrystalline polymer which its degree of crystallinity could not be measured directly. This is due to the fact that the very thin layer

Table 3 Preparation conditions and permeation performance of confirmation experiments

\begin{tabular}{|c|c|c|c|c|c|c|c|c|}
\hline $\begin{array}{l}\text { Confirmation } \\
\text { tests }\end{array}$ & $\begin{array}{l}\text { MPD } \\
\text { concentration } \\
(\mathrm{wt} \%)\end{array}$ & $\begin{array}{l}\text { TMC } \\
\text { concentration } \\
\text { (wt } \%)\end{array}$ & $\begin{array}{l}\text { Reaction } \\
\text { time (s) }\end{array}$ & $\begin{array}{l}\text { Curing } \\
\text { temperature } \\
\left({ }^{\circ} \mathrm{C}\right)\end{array}$ & $\begin{array}{l}\text { Average } \\
\text { water flux } \\
(\mathrm{LMH})\end{array}$ & $\begin{array}{l}\text { Taguchi } \\
\text { predicted } \\
\text { flux (LMH) }\end{array}$ & $\begin{array}{l}\text { Average salt } \\
\text { rejection } \\
(\%)\end{array}$ & $\begin{array}{l}\text { Taguchi } \\
\text { predicted } \\
\text { rejection (\%) }\end{array}$ \\
\hline C1 & 1 & 0.15 & 15 & 55 & 20.9 & 42.8 & 96.9 & 95.7 \\
\hline $\mathrm{C} 2$ & 2 & 0.15 & 15 & 55 & 19.6 & 12.8 & 97.4 & 98 \\
\hline C5 & 1 & 0.15 & 15 & 85 & 15.6 & 31 & 97.5 & 96.4 \\
\hline C6 & 2 & 0.15 & 30 & 55 & 19.4 & 13.3 & 97.6 & 97.8 \\
\hline C7 & 3 & 0.15 & 15 & 55 & 110.5 & - & 72.1 & - \\
\hline C8 & 3 & 0.35 & 15 & 55 & 30.6 & - & 96.7 & - \\
\hline C9 & 1 & 0.25 & 15 & 55 & 46.0 & 56 & 95.2 & 94.6 \\
\hline C10 & 2 & 0.25 & 15 & 55 & 26.0 & 25 & 96.4 & 97 \\
\hline
\end{tabular}



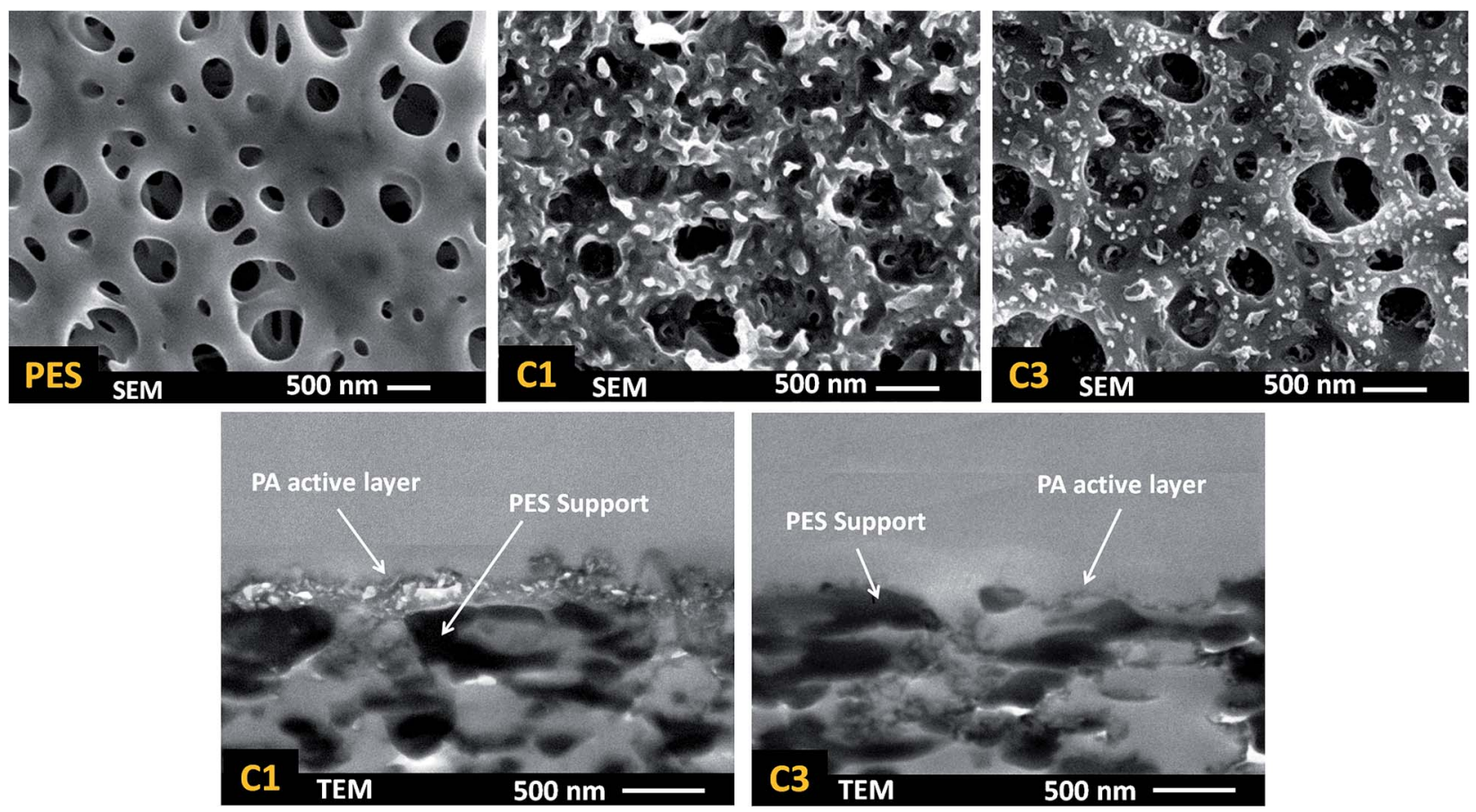

Fig. 5 FESEM surface and TEM cross-section images of confirmation test membranes: PES support, C1 (MPD 1 wt\%, TMC 0.15 wt\%) and C3 (MPD $1 \mathrm{wt} \%, \mathrm{TMC} 0.35 \mathrm{wt} \%)$ to study the effect of TMC concentration. The levels of other parameters are presented in Table 3.

of PA, synthesized by in situ polymerization, is not detachable from the PES support to be used for further tests on crystallinity. Therefore, in the present work, the discussions on the density and/or crystallinity of the membranes are provided based on the pure water flux and salt rejection results.

It was shown by Freger ${ }^{31}$ that the cross-link density of the PA film is not uniform across the cross-section where the incipient thin film is proposed to be the densest and actual selective layer over the whole structure. ${ }^{31,32}$ Since the monomer diffusion and reaction rate in the slow growth stage strongly depend on the mass transfer resistance of the core layer, any factor which changes the properties of this layer (in terms of the thickness and cross-link density) will affect the final morphology and separation performance of the resulting films.

Regarding the Fig. 3a, the flux decline with the increase in MPD concentration could be attributed to the increase in the overall thickness of the PA film during the course of polymerization. At higher MPD concentration, there will be a larger driving force for MPD to diffuse through the initially formed PA film. The increase in the number of available MPD molecules in the reaction zone promotes polymerization, thus increases the

Table 4 Atomic concentration of $\mathrm{C}, \mathrm{O}$ and $\mathrm{N}$ in PA layer of $\mathrm{C} 1$ and $\mathrm{C} 3$ membranes obtained by XPS analysis

\begin{tabular}{llrlll}
\hline & \multicolumn{3}{l}{ Atomic concentration (\%) } & \\
\cline { 2 - 5 } Sample & O 1s & N 1s & C 1s & S 2p & $\begin{array}{l}\text { Cross-linking } \\
\text { density (\%) }\end{array}$ \\
\hline C1 & 14.74 & 9.03 & 75.97 & 0.25 & 28 \\
C3 & 12.75 & 10.31 & 76.72 & 0.23 & 69
\end{tabular}

membrane internal resistance toward water passage by forming a thicker PA film with bigger ridge-and-valley structure at the surface. ${ }^{13}$

The observed trend for the influence of the TMC concentration was also confirmed by comparing the permeation flux of confirmation membranes C1 vs. C3 and C2 vs. C4 as presented in Table 3.

It is a widely held view that in a typical IP reaction, an increase in the TMC concentration decreases the amine to acyl chloride molar ratio $\left(-\mathrm{NH}_{2} /-\mathrm{COCl}\right)$ and results in a thinner but denser PA active film with lower water flux in overall. ${ }^{13,32}$ Surface images of the membranes made at high TMC concentration (C3) in this study also confirmed the formation of a thin and dense film at the surface (based on more resemblance of PES and C3 surface morphology). However, significant increase in the water flux with increasing in TMC concentration (from 20.9 $\mathrm{LMH}$ in $\mathrm{C} 1$ to $79.1 \mathrm{LMH}$ in $\mathrm{C} 3$ ), not predicted by the above hypothesis, reveals the complex influence of the film thickness and density on the transport properties of membranes.

The thickness and cross-link density of PA layer were measured by TEM and XPS analyses, respectively to approve above discussion. The cross-linking density of the whole PA active layer (not merely the incipient layer) can be obtained with the following equation: ${ }^{33}$

$$
\text { Cross-linking density }=\frac{m}{m+n} \times 100
$$

where $m$ and $n$ are the cross-linked and linear part of polyamide network. According to chemical formula of fully cross-linked $\left(\mathrm{C}_{18} \mathrm{H}_{12} \mathrm{~N}_{3} \mathrm{O}_{3}\right)$ and fully linear $\left(\mathrm{C}_{15} \mathrm{H}_{10} \mathrm{O}_{4} \mathrm{~N}_{2}\right)$ polyamide, the theoretical $\mathrm{O} / \mathrm{N}$ ratio can be presented by: ${ }^{33}$ 


$$
\frac{\mathrm{O}}{\mathrm{N}}=\frac{3 m+4 n}{3 m+2 n}
$$

Using eqn (5), the value of $m$ and $n$ can be calculated based on the $\mathrm{O} / \mathrm{N}$ ratio obtained from XPS data. For example, for the C1 and C3 membranes shown in Fig. 5, the XPS results are presented in Table 4 . As can be seen, the cross-linking density of C1 and C3 are 28\% and 69\%, respectively, which confirms our hypothesis about the higher density of C3 membrane. However, it must be noted that, the density of the core layer, which is commonly used to justify conclusions (e.g. change in surface morphology) cannot be measured with characterization tests because it is covered by the outer loose layer. In the present study, we qualitatively discussed and compared the density of core layer for different membranes by noting that the mass transfer resistance of the incipient layer to MPD diffusion is directly related to its density. As mentioned earlier, in the case of denser core layer, the mass transfer resistance against diffusion of MPD diffusion is larger and thus less ridge-andvalley morphology forms on the surface.

The surface FESEM and cross-section TEM images of two confirmation test membranes along with PES substrate are shown in Fig. 5. Unlike microporous PES supports which have a relatively smooth and featherless morphology, C1 had a tortuous and ridge-and-valley structure at its surface. ${ }^{25}$ However, by increasing the TMC concentration from $0.1 \mathrm{wt} \%$ (C1) to 0.35 wt\% (C3), the morphology changed to a nodular structure with small microprotuberances at the surface. This again confirms formation of a denser PA core layer which hindered diffusion of MPD, thereby prevented film growth and formation of ridge-and-valley features on the surface. Moreover, the TEM images illustrate that increasing the TMC concentration results in a marked decrease in PA film thickness which is highly desirable for reducing the hydraulic resistance of membrane against water permeation.

The substantial change in surface morphology and the absence of ridge-and-valley structure at the surface by increasing TMC concentration is a proof of concept for formation of a thin and dense core layer at the early stage of IP reaction. At higher TMC concentration, due to the presence of a higher number of TMC molecules in the reaction zone, the incipient PA layer became dense enough that hindered MPD molecules to diffuse to organic phase and form a thick ridgeand-valley structure during the time of IP reaction. There are two likely causes for such a significant increase in permeation flux by increasing the TMC concentration: first, there is a marked decrease in the thickness of the PA active layer as it is shown by TEM images in Fig. 5, which decreases membrane resistance against mass transfer and eventually increases the water flux. Second, a thinner active layer is more prone to be affected by the subsurface morphology (e.g. porosity and roughness of the support) than the thicker ones, the presence of any open nanopores at the substrate due to incomplete coverage by the thin film may manifest themselves by increasing the water flux and lowering the salt rejection.
The typical trade-off behaviour between salt rejection and water flux was also observed in confirmation experiments. According to Table 3, increasing TMC concentration from $0.1 \mathrm{wt} \%$ (C1) to $0.35 \mathrm{wt} \%$ (C3) increased water flux significantly (almost four times), but decreased the salt rejection slightly from $96.9 \%$ to $91.4 \%$. The salt rejection results from the confirmation experiments (C1-C4) show the dramatic role of monomers concentration. The range of results varies from high rejection/low flux RO membranes to low rejection/high flux NF membranes.

\section{Effect of curing temperature}

Heat curing is primarily performed to speed up evaporation of both water and organic solvent from the membrane surface and to help termination of the polymerization reaction. ${ }^{\mathbf{1 0}}$ The average effect of curing temperature on the performance of TFC membranes is presented in Fig. $3 \mathrm{c}$ and 4c. According to these figures, curing at room temperature $\left(25^{\circ} \mathrm{C}\right)$ resulted in TFC membranes with a high rejection but poor water flux. Raising the temperature to $55^{\circ} \mathrm{C}$ increased the flux roughly 5 times with only a $2 \%$ reduction in rejection. Further increase in the curing temperature slightly decreased the flux and improved the rejection. This result is confirmed by comparing the performance of base membrane M1 (Table 2) and by confirmation experiments $\mathrm{C} 1$ and $\mathrm{C5}$ (Table 3). The observed variation in water permeation and salt rejection of the membranes can be justified by monitoring the change in surface morphology at various curing temperatures. The surface images of M1, C1 and C5 (Fig. 6) indicates that the PA film underwent a surface densification with increasing curing temperature. The thin earlike PA ridges at the surface of $\mathrm{M} 1$ (cured at $25{ }^{\circ} \mathrm{C}$ ) merged together and became larger in $\mathrm{C} 1$ (cured at $55^{\circ} \mathrm{C}$ ). This change in morphology increased water permeation from 7.1 LMH in M1 to $20.9 \mathrm{LMH}$ in $\mathrm{C} 1$. However, further increase in the curing temperature to $85{ }^{\circ} \mathrm{C}$ (above the boiling point of cyclohexane) resulted in film shrinkage due to the fast evaporation of solvent residue from the $\mathrm{C} 5$ membrane which could explain the slight decrease in the water flux for this membrane. ${ }^{\mathbf{1 0}, 13}$

\section{Effect of reaction time}

Fig. $3 \mathrm{~d}$ and $4 \mathrm{~d}$ present the average effect of the reaction time on the transport properties of the TFC membranes. It can be observed that changing the polymerization time from $15 \mathrm{~s}$ to $60 \mathrm{~s}$ did not result in an appreciable change in the average flux and rejection. The observed trend was also verified by the performance results of confirmation membranes $\mathrm{C} 2$ and $\mathrm{C} 6$ (Table 3) and base membrane M7. A very narrow range of water flux (19.4-19.9 LMH) and salt rejection (97.4-97.7\%) indicated a trivial effect of reaction time on the membrane properties.

Based on the IP reaction mechanism, prolonging the reaction time should increase film thickness and thus membrane resistance to water flow. However, a negligible shift in flux and rejection with the change in reaction time reveals that the growth of the PA film after $15 \mathrm{~s}$ was insignificant and implies that the permeation was mainly controlled by the dense core layer which was rapidly formed at the surface. 

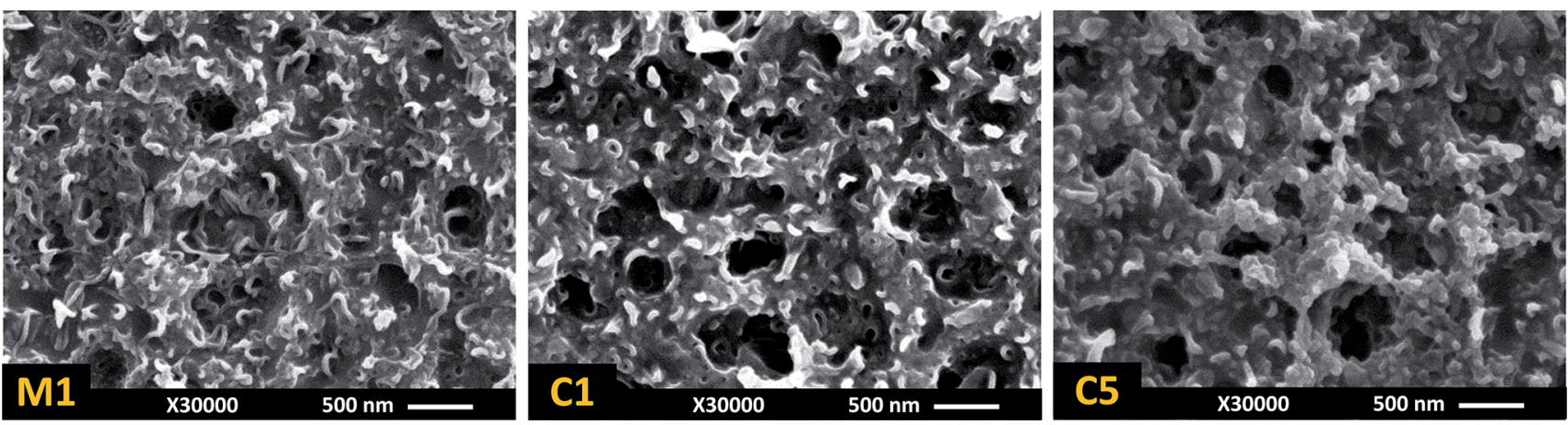

Fig. 6 FESEM surface morphologies of Taguchi base membrane $\mathrm{M} 1$ (cured at $25^{\circ} \mathrm{C}$ ) and confirmation test membranes $\mathrm{C} 1$ (cured at $55^{\circ} \mathrm{C}$ ) and $\mathrm{C} 5$ (cured at $85^{\circ} \mathrm{C}$ ) to study the effect of curing temperature. The levels of other parameters are presented in Table 3.

\section{Interaction between MPD and TMC concentration}

The interaction between two factors, in a simple explanation, accounts for a condition where a factor shows different behaviour at different levels of the other factor. ${ }^{18}$ Identifying the possible interactions between synthesis parameters can be quite helpful as it not only improves the basic understanding of the PA formation via interfacial polymerization reaction, but also increases the efficiency and output quality of the preparation process. Although exploiting saturated orthogonal arrays (like L9) eliminates the chance of extracting the interactions directly from OA table, it can provide guidance to track probable interactions between influential parameters. According to Fig. 3 and 4, the average trends of the influence of TMC and MPD concentrations are in opposite directions, which suggest a possible interaction between these two factors. The interaction between two factors can be visually represented by plotting the impact of one of these factors on the response at different level of the other factor. In general, an interaction exists when the straight lines are not parallel. ${ }^{18}$ Employing the water flux data in Table 3, a two way interaction plot for the TMC and MPD concentrations is presented in Fig. 7. The intersecting lines in this figure reveal the existence of a mutual interaction between the TMC and MPD concentrations.

Fig. 7 shows that MPD concentration induced different effects on permeation flux when TMC changed from $0.15 \mathrm{wt} \%$ to $0.35 \mathrm{wt} \%$ in organic solution. At MPD concentration of $1 \mathrm{wt} \%$, the water flux significantly improved from 20.9 LMH (C1) to 79.1 LMH (C3) with the increase in TMC concentration. When MPD concentration in aqueous phase increased to $2 \mathrm{wt} \%$, the rate of flux enhancement decreased, which supports the accepted hypothesis that membranes become thicker with higher internal resistance at higher MPD concentrations. However, at $3 \mathrm{wt} \% \mathrm{MPD}$, the trend was different: water flux first dropped dramatically from 110.5 LMH in $\mathrm{C} 7$ to $15.3 \mathrm{LMH}$ in $\mathrm{C} 11$, then increased to $30.6 \mathrm{LMH}$ in $\mathrm{C} 8$ with further increase in the TMC concentration.

The SEM images of C2, C4, C7 and C8 membranes (Fig. 8) provide clear evidence that the unexpected shift in water flux was the result of a significant change in the surface structure of the membranes. The $\mathrm{C} 7$ membrane, prepared at high MPD (3 wt $\%$ ) and low TMC (0.15 wt\%) concentrations, had completely different morphology from previously seen ridgeand-valley and nodular structures. The markedly thick and fully formed PA film at the surface of this membrane reveals that due to the lack of sufficient TMC molecules, the incipient core layer was not dense enough to slow down the polymer growth at the surface. Therefore, rapid diffusion of MPD molecules to the reaction zone in the organic phase resulted in a thick but loose structure with high water flux and low salt rejection. By increasing the TMC concentration to $0.35 \mathrm{wt} \%$ in $\mathrm{C} 8$, the active layer became dense again and the surface morphology transformed to a nodular structure, similar to C3 and $\mathrm{C} 4$ membranes which were similarly prepared at high TMC concentration.

According to the interaction plot (Fig. 7), it can be concluded the widely accepted view that the lower amine/acyl chloride molar ratio (closer to unity) results in a denser polyamide film with lower water flux, ${ }^{13,28,32,34}$ cannot be simply generalized to all practices. The flux and rejection of the PA films is directly related to the surface structure and more specifically to the cross-link density of the core layer which are controlled by initial concentration of both monomers at the beginning of IP

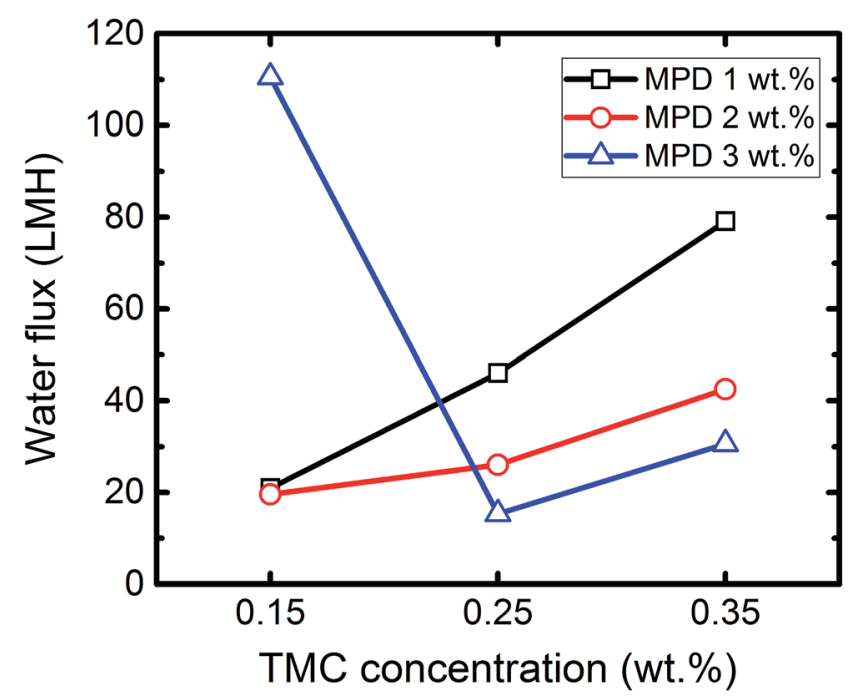

Fig. 7 Two-way interaction plot for MPD and TMC concentration (reaction time: $15 \mathrm{~s}$, curing temperature: $55^{\circ} \mathrm{C}$ ). 
reaction rather than a single molar ratio. The very high (31.6 in C7) and low (4.51 in C2) molar ratio in our experiments resulted in high flux membranes at the expense of salt rejection decline, suggesting that there is an acceptable range of monomer concentration which yields salt rejecting $\mathrm{NF}$ and $\mathrm{RO}$ membranes with desirable water flux $(>90 \%)$. In order to make TFC membranes with tailored properties, this range must be first identified by considering the monomer concentration interaction at very high and low molar ratios.

\section{Analysis of variance (ANOVA)}

When manipulating this series of experimental data, it was informative to determine the contribution weight of different factors and to recognize the most influential one(s). ANOVA is a useful statistical method to determine the contribution and significance of each parameter to the total variation of the results. This analysis starts with calculation of the total $\left(\mathrm{SS}_{\mathrm{T}}\right)$ and factor sum of squares $\left(\mathrm{SS}_{A}\right)$ using the following equations: ${ }^{18}$

$$
\begin{gathered}
\mathrm{SS}_{\mathrm{T}}=\sum_{i=1}^{N} Y_{i}^{2}-\frac{T^{2}}{N} \\
\mathrm{SS}_{A}=\sum_{i=1}^{K_{A}}\left(\frac{A_{i}^{2}}{n_{A i}}\right)-\frac{T^{2}}{N}
\end{gathered}
$$

where $Y_{i}$ is an experimental result (observation), $A_{i}$ is the sum of observations at level $i$ of factor $A$, and $T$ is the sum of all observations. $K_{A}$ is the number of levels of factor $A\left(K_{A}=3\right.$ for all factors in the present study), $n_{A i}$ is number of experiment involving level $i$ of factor $A$ ( $n_{A i}=6$ in the present study) and $N$ is the total number of experiments (18 in this work). The error sum of squares $\left(\mathrm{SS}_{\mathrm{e}}\right)$ can be calculated as:

$$
\mathrm{SS}_{\mathrm{e}}=\mathrm{SS}_{\mathrm{T}}-\left(\mathrm{SS}_{A}+\mathrm{SS}_{B}+\ldots\right)
$$

where $A$ and $B$ are different controllable factors. It is worth mentioning that the error term here not only accounts for experimental error, but also represents the effect of all other factors (like soaking and drying conditions and curing time) which are not considered in the experiments. The variance of factor $A$ can be obtained by dividing the sum of squares by the degree of freedom, $V_{A}=\mathrm{SS}_{A} / \mathrm{DOF}_{A}$, where $\mathrm{DOF}_{A}=K_{A}-1$. Similarly, the variance of error can be calculated by $V_{\mathrm{e}}=$ $\mathrm{SS}_{\mathrm{e}} / \mathrm{DOF}_{\mathrm{e}}$ where $\mathrm{DOF}_{\mathrm{e}}$ is the error degree of freedom and is given by $\mathrm{DOF}_{\mathrm{e}}=\mathrm{DOF}_{\mathrm{T}}-\left(\mathrm{DOF}_{A}+\mathrm{DOF}_{B}+\ldots\right)$. In this equation, $\mathrm{DOF}_{\mathrm{T}}$ is the total degree of freedom and is equal to $N-1$.

The ratio of factor variance to error variance (known as $F$-ratio) is an important statistical parameter, which is used to estimate the significance of a factor. It is calculated as follows:

$$
F_{A}=\frac{V_{A}}{V_{\mathrm{e}}}
$$

If the calculated $F$-ratio for a factor is larger than the extracted $F$ from table ( $F$-table can be obtained from statistical tables using $\mathrm{DOF}_{A}$ and $\mathrm{DOF}_{\mathrm{e}}$ for different risks, $\alpha$ ), it can be concluded that the influence of the factor on the results is significant and cannot be ignored. In fact, it shows that the change in a response is due to the alteration of the level of that factor and not because of noise factors. Table 5 presents the
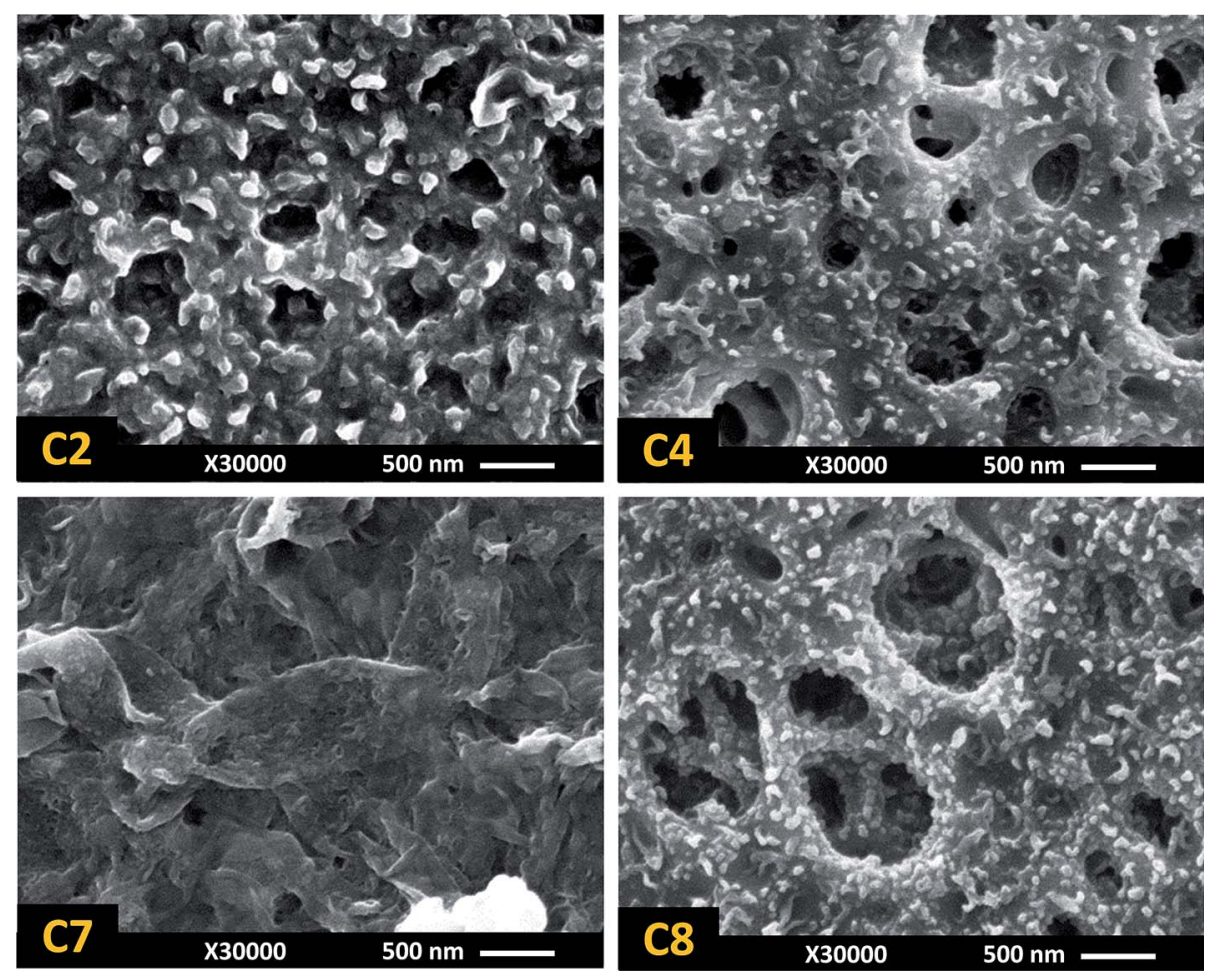

Fig. 8 Surface images of confirmation membranes C2 \& C4 (MPD concentration 2 wt\%) and C7 \& C8 (MPD concentration 3 wt\%). The microporous structure of PES support is clear for C7 and C8 due to formation of thin dense layer at the surface. 
Table 5 Analysis of variance based on water permeation data

\begin{tabular}{lrllrc}
\hline Factor & $\mathrm{SS}^{a}$ & $\mathrm{DOF}^{b}$ & Variance & $F$-ratio & $P^{d}(\%)$ \\
\hline Curing temperature $\left({ }^{\circ} \mathrm{C}\right)$ & 3977 & 2 & 1988 & 111.0 & 40.7 \\
MPD concentration (\%) & 2733 & 2 & 1366 & 76.3 & 28.0 \\
TMC concentration (\%) & 2720 & 2 & 1360 & 75.9 & 27.8 \\
Reaction time (s) & 187 & 2 & 94 & 5.2 & 1.9 \\
Error (\%) & 161 & 9 & 18 & - & -
\end{tabular}

${ }^{a}$ Sum of squares. ${ }^{b}$ Degree of freedom. ${ }^{c} F$-table for $\alpha$ (risk) $=0.05$ is $F=4.26 .{ }^{d}$ Percent contribution of each factor on response $\left(\mathrm{SS}_{A} / \mathrm{SS}_{\mathrm{T}}\right)$.

analysis of variance of experimental results based on water flux data. According to this table, the calculated $F$-ratio for all factors was greater than the extracted $F$-table $(F=4.26)$ with $95 \%$ confidence $(\alpha=0.05)$ which means all factors had significant effect on the response.

The percent contribution of each factor $(P)$ can also be evaluated by considering the sum of squares values using the equation $P_{A}=\mathrm{SS}_{A} / \mathrm{SS}_{\mathrm{T}} \times 100$. According to the $P$ values presented in Table 5, curing temperature had the highest contribution $(40.7 \%)$ to the variance in the water permeation in comparison to other factors. The MPD and TMC concentrations had similar contribution to permeate flux, $28 \%$ and $27.8 \%$, respectively. Reaction time, as was observed in the marginal plots (Fig. 2), had the minimum contribution (1.9\%) on the response. It must be noted that the extracted contribution values are limited to the levels of control factors considered in this study and could be different for a different set of factor combinations.

Using the Taguchi method, a prediction model (transfer function) is developed by the following equation to predict the performance of TFC membranes at the levels which are not considered in the L9 table. ${ }^{35}$

$$
\text { Response }=\overline{\mathrm{MPD}_{i}}+\overline{\mathrm{TMC}_{j}}+\overline{\text { Reaction }_{k}}+\overline{\text { Curing }_{l}}-3 \times \frac{T}{N}
$$

where response can be water flux or salt rejection and $\overline{\mathrm{MPD}_{i}}$, $\overline{\mathrm{TMC}_{i}}, \overline{\text { Reaction }_{k}}$, and $\overline{\text { Curing }_{l}}$ are the average values of observations (flux or rejection) for MPD and TMC concentration, reaction time and curing temperature, respectively at $i, j, k$ and $l$ levels of these variables. These average values are presented in Fig. 3 and 4 . This model exploits a linear interpolation between the levels of controllable factors. The predicted flux and rejection are presented in Table 3. Note that the prediction data for C7, C8 and C11 membranes were not presented as the monomers concentration for these trials were not within the range of initial levels. As can be seen in Table 3, the Taguchi prediction model predicts the permeation flux pretty well for the high flux membranes ( $\mathrm{C} 3$ and $\mathrm{C} 4$ ) where the monomers concentration, particularly TMC, are set to the highest level. However, it fails to precisely predict the flux of $\mathrm{C} 1$ and $\mathrm{C} 5$ membranes which are prepared at the lowest level of TMC and MPD concentration. The relatively high prediction error for the flux of C1 and C5 membranes can be due to simplicity of the prediction model, as it assumes that the change in the results follows a linear behavior. From the other point of view, it can be indicative of complexity and nonlinearity of film formation by IP reaction when the TMC concentration in the organic solution is low $(0.15$ $\mathrm{wt} \%)$. However, as the TMC concentration increases ( $0.35 \mathrm{wt} \%)$, the model prediction and experimental results match very well, suggesting a more predictable mechanism for the polymerization reaction at the surface.

\section{Conclusions}

The effects of simultaneous change in four synthesis factors: MPD and TMC concentration, reaction time and heat curing temperature on the transport properties of interfacially polymerized PA TFC membranes were systematically investigated using the Taguchi method (L9 orthogonal arrays). Plotting the "marginal means" of control factors showed that the average influence of increasing the MPD concentration was to lower water flux and to improve salt rejection, whereas increasing the TMC concentration resulted in more permeable membranes with lower rejection. Furthermore, a strong interaction was found between MPD and TMC concentrations, suggesting the importance of monomer concentration (or molar) ratio rather than on individual concentration. The analysis of variance (ANOVA) revealed that the water flux through synthesized membranes was most dramatically affected by curing temperature (40\%). The MPD and TMC concentration also had significant effect ( $28 \%$ and $27.8 \%$, respectively). Changing the reaction time in the range from $15 \mathrm{~s}$ to $60 \mathrm{~s}$ was found to have the lowest influence on water permeation (1.9\%). This study shows the significant role of the four considered synthesis parameters on the final properties of TFC membranes and these results can be used for making NF and RO membranes with a wide range of water flux and salt rejection characteristics.

\section{References}

1 M. A. Shannon, P. W. Bohn, M. Elimelech, J. G. Georgiadis, B. J. Mariñas and A. M. Mayes, Nature, 2008, 452, 301-310.

2 D. Li and H. Wang, J. Mater. Chem., 2010, 20, 4551.

3 N. Misdan, W. J. Lau and A. F. Ismail, Desalination, 2012, 287, 228-237.

4 K. P. Lee, T. C. Arnot and D. Mattia, J. Membr. Sci., 2011, 370, 1-22.

5 G.-Y. Chai and W. B. Krantz, J. Membr. Sci., 1994, 93, 175192.

6 A. P. Rao, N. V. Desai and R. Rangarajan, J. Membr. Sci., 1997, 124, 263-272.

7 A. P. Rao, S. V. Joshi, J. J. Trivedi, C. V. Devmurari and V. J. Shah, J. Membr. Sci., 2003, 211, 13-24.

8 Y. Song, P. Sun, L. Henry and B. Sun, J. Membr. Sci., 2005, 251, 67-79.

9 I. J. Roh, A. R. Greenberg and V. P. Khare, Desalination, 2006, 191, 279-290.

10 A. K. Ghosh, B.-H. Jeong, X. Huang and E. M. V. Hoek, J. Membr. Sci., 2008, 311, 34-45.

11 M. Liu, S. Yu, J. Tao and C. Gao, J. Membr. Sci., 2008, 325, 947-956. 
12 Y. Jin and Z. Su, J. Membr. Sci., 2009, 330, 175-179.

13 W. Xie, G. M. Geise, B. D. Freeman, H.-S. Lee, G. Byun and J. E. McGrath, J. Membr. Sci., 2012, 403-404, 152-161.

14 C. Klaysom, S. Hermans, A. Gahlaut, S. Van Craenenbroeck and I. F. J. Vankelecom, J. Membr. Sci., 2013, 445, 25-33.

15 V. Czitrom, Am. Stat., 2012, 53, 126-131.

16 D. D. Frey, F. Engelhardt and E. M. Greitzer, Res. Eng. Des., 2003, 14, 65-74.

17 M. Sadrzadeh, A. Razmi and T. Mohammadi, Sep. Purif. Technol., 2007, 54, 147-156.

18 R. K. Roy, Design of Experiments Using The Taguchi Approach: 16 Steps to Product and Process Improvement, John Wiley \& Sons, Inc., 2001.

19 M. Sadrzadeh, J. Hajinasiri, S. Bhattacharjee and D. Pernitsky, Sep. Purif. Technol., 2015, 141, 339-353.

20 M. Mulder, Basic Principles of Membrane Technology, Springer, Netherlands, Dordrecht, 1996.

21 B. C. Smith, Infrared Spectral Interpretation: A Systematic Approach, CRC Press, 1998.

22 C. Tang, Y. Kwon and J. Leckie, J. Membr. Sci., 2007, 287, 146156.
23 P. W. Morgan and S. L. Kwolek, J. Polym. Sci., 1959, 40, 299327.

24 J. E. Cadotte, R. J. Petersen, R. E. Larson and E. E. Erickson, Desalination, 1980, 32, 25-31.

25 R. J. Petersen, J. Membr. Sci., 1993, 83, 81-150.

26 J. E. Cadotte, in Material Science of Synthetic Membranes, ACS Publications, 1985, pp. 273-294.

27 H. Yan, X. Miao, J. Xu, G. Pan, Y. Zhang, Y. Shi, M. Guo and Y. Liu, J. Membr. Sci., 2015, 475, 504-510.

28 A. V. Berezkin and A. R. Khokhlov, J. Polym. Sci., Part B: Polym. Phys., 2006, 44, 2698-2724.

29 R. W. Baker, Membrane Technology and Applications, John Wiley \& Sons, Chichester, UK, 3rd edn, 2012.

30 B. S. Lalia, V. Kochkodan, R. Hashaikeh and N. Hilal, Desalination, 2013, 326, 77-95.

31 V. Freger, Langmuir, 2003, 19, 4791-4797.

32 V. Freger and S. Srebnik, J. Appl. Polym. Sci., 2003, 88, 11621169.

33 O. Akin and F. Temelli, Desalination, 2011, 278, 387-396.

34 V. Freger, Langmuir, 2005, 21, 1884-1894.

35 M. Amirilargani, M. Sadrzadeh and T. Mohammadi, J. Polym. Res., 2009, 17, 363-377. 